\title{
Comparing Face-to-Face and Online Data Collection Methods in Preterm and Full-Term Children: An Exploratory Study
}

\author{
Paige M. Nelson ${ }^{1 *}$, Francesca Scheiber ${ }^{1}$, Haley M. Laughlin ${ }^{1}$ and Ö. Ece Demir-Lira ${ }^{1,2,3}$ \\ ${ }^{1}$ Department of Psychological and Brain Sciences, The University of lowa, lowa City, IA, United States, ${ }^{2}$ Delta Center, \\ The University of lowa, lowa City, IA, United States, ${ }^{3}$ lowa Neuroscience Institute, The University of lowa, lowa City, IA, \\ United States
}

OPEN ACCESS

Edited by:

Lisa Oakes,

University of California, Davis,

United States

Reviewed by:

Rachel M. Flynn,

San Francisco State University,

United States

Cristina de-la-Peña

Universidad Internacional de La Rioja,

Spain

*Correspondence:

Paige M. Nelson

paige-nelson@uiowa.edu

Specialty section: This article was submitted to

Developmental Psychology,

a section of the journal

Frontiers in Psychology

Received: 30 June 2021 Accepted: 08 October 2021 Published: 28 October 2021

Citation:

Nelson PM, Scheiber F, Laughlin HM and Demir-Lira ÖE

(2021) Comparing Face-to-Face and Online Data Collection Methods in Preterm and Full-Term Children: An

Exploratory Study.

Front. Psychol. 12:733192.

doi: 10.3389/fpsyg.2021.733192
The COVID-19 pandemic has transformed the landscape for children's daily lives and the landscape for developmental psychology research. Pandemic-related restrictions have also significantly disrupted the traditional face-to-face methods with which developmental scientists produce research. Over the past year, developmental scientists have published on the best practices for online data collection methods; however, existing studies do not provide empirical evidence comparing online methods to faceto-face methods. In this study, we tested feasibility of online methods by examining performance on a battery of standardized and experimental cognitive assessments in a combined sample of 4- to 5-year-old preterm and full-term children, some of whom completed the battery face-to-face, and some of whom completed the battery online. First, we asked how children's performance differs between face-to-face and online format on tasks related to verbal comprehension, fluid reasoning, visual spatial, working memory, attention and executive functioning, social perception, and numerical skills. Out of eight tasks, we did not find reliable differences on five of them. Second, we explored the role of parent involvement in children's performance in the online format. We did not find a significant effect of parent involvement on children's performance. Exploratory analyses showed that the role of format did not vary for children at risk, specifically children born preterm. Our findings contribute to the growing body of literature examining differences and similarities across various data collection methods, as well as literature surrounding online data collection for continuing developmental psychology research.

Keywords: COVID-19, in-person data collection, online data collection, children, prematurity, neurocognitive assessment, developmental psychology

\section{INTRODUCTION}

The COVID-19 pandemic has transformed the landscape for children's daily lives and the landscape for developmental psychology research. Schools across the world have restructured and developed online learning curriculums. Around 214 million children are estimated to have missed more than three quarters of in-person education in 2020 (United Nations Children's Fund, 2021). More than $90 \%$ of children in the United States are estimated to have received some form of distance learning 
during COVID-19 (Bureau, 2021). Likewise, the provision of community and social services has suffered, despite being needed now more than ever (Tsega et al., 2020; EducationData, 2021). Pandemic-related restrictions have also significantly disrupted the traditional methods in which developmental scientists produce research-that is, in-person studies requiring face-toface interactions (Garrisi et al., 2020). Given the likely continued role of online assessments in research and clinical services, it is important to understand both the differences and similarities between children's performance in face-to-face and online settings. The direct and long-term effects of online measurement of children's performance remain largely unknown. The goal of the current paper is to add to this growing body of literature by testing the feasibility of online data collection methods and comparing 4- to 5-year-old preschoolers' performance face-toface vs. online on a wide variety of standardized and experimental cognitive assessments.

Over the past few years, developmental scientists have published on the best practices for online data collection methods (Frank et al., 2016; Garrisi et al., 2020; Lourenco and Tasimi, 2020; Manning et al., 2020; Nussenbaum et al., 2020; Rhodes et al., 2020; Sheskin et al., 2020; Morini and Blair, 2021; Su and Ceci, 2021). These studies agree that while online data collection methods are still in their infancy, online measurements have become a promising platform for developmental psychology research. However, only a few studies provide empirical evidence comparing online methods to face-to-face methods. For example, Morini and Blair (2021) examined the feasibility of collecting remote eye-gaze data with children. They compared their online sample to a previously collected face-to-face sample, during which they found their online data collection methods to be reliable and sufficient in conducting developmental language research (Morini and Blair, 2021).

An emerging body of work also focuses on the reliability and validity of online data collection methods. For example, Manning et al. (2020) examined the feasibility, reliability, and validity of child language samples drawn from recorded parent-child interactions via video chat. They found child language samples (i.e., key speech and language measures) collected via video chat vs. face-to-face laboratory video recordings to be comparable (Manning et al., 2020). So far, studies have focused on narrow aspects of children's performance, thus it is important to examine children's performance on a wide array of standardized and experimental measures assessing multiple cognitive domains to gain a more complete view of face-to-face vs. online assessments. Our primary goal is to examine how children's performance in verbal comprehension, fluid reasoning, visual spatial, working memory, attention and executive functioning, social perception, and numerical tasks differ between a lab based, face-to-face format, and an online format.

According to some, remote research has many benefits, including its ability to broaden sample diversity, as compared to face-to-face laboratory studies (Lourenco and Tasimi, 2020). For example, online methods might be more inclusive of groups who do not or cannot attend face-to-face research studies, including atypically developing children. Given the greater need for assessments and interventions for at-risk children in clinical settings, it is fundamental to better understand whether and how online interactions influence children with atypical developmental trajectories. Children born preterm $(<37$ weeks gestational age) fall into such an "at risk" group. Every year, close to 15 million children in the United States are born preterm (Wolke et al., 2019). Those who survive have an increased risk for death, disability, and delay (Centers for Disease Control and Prevention [CDC], 2020). Preterm-born children (PTB) fall behind term-born children (TB) on various measures of cognitive performance (Allotey et al., 2018; Brydges et al., 2018), with children born the earliest tending to have the worst outcomes (Bhutta et al., 2002; Snijders et al., 2020). Likewise, this gap in cognitive performance between PTB and TB children often persists throughout formal schooling. Better understanding how at-risk children perform in face-to-face vs. online formats will have implications for future assessment and intervention efforts. Thus, in the current study, we diversify our sample by including both PTB children and TB children.

Taken together, our goal is to contribute to the growing literature on establishing the reliability of online research methods by examining children's performance on standardized and experimental cognitive assessments. We examine performance in a combined sample of 4- to 5-year-old TB and PTB children, some of whom completed the battery faceto-face and some of whom completed the battery online. We ask how children's performance differs between face-to-face and online format on tasks related to verbal comprehension, fluid reasoning, visual spatial, working memory, attention and executive functioning, social perception, and numerical skills. We supplement our main research question with two exploratory analyses. Online data collections methods typically rely on parents, but how parent involvement during remote data collection influences children's performance has yet to be explored. To address this question, we explore the role of parent involvement in children's performance in the online format. How children's performance differs between face-to-face and online format on tasks related to verbal comprehension, fluid reasoning, visual spatial, working memory, attention and executive functioning, social perception, and numerical skills as a function of a prematurity also has yet to be explored. To address this question as an exploratory aim, we examine the role of prematurity in children's performance in both the face-to-face and online format.

\section{METHODS}

\section{Participants}

Participants were $93 \mathrm{~TB}$ ( $\geq 37$ weeks gestational age) and 38 PTB ( $<37$ weeks gestational age), for a total of 131 children, who participated in an ongoing longitudinal study on the relations between preterm birth and neurodevelopment. Fifty-four TB children and 29 PTB completed the study face-to-face in a labbased format. Thirty-nine TB children and nine PTB completed the study in an online format via Zoom video conferencing. Overall, 83 children (29 PTB) completed the study face-to-face in a lab-based format and 48 children (9 PTB) completed the study in an online format. This study was approved by the Institutional Review Board at our local university. We recruited parent-child 
dyads using the university hospital's electronic health records, university mass emailing, social media, and word of mouth. Parent-child dyads were eligible for this study if the child was between the ages of 4 and 5 years old, was a native speaker of English, had normal or corrected-to-normal vision and hearing, had no history of a genetic syndrome or birth defect, and had no limitations (based on parental report) that would prevent them from completing paper/pencil tasks. For those who completed the study in an online format, it was also preferred that they had an electronic device (computer, laptop, tablet, or smart phone) with reliable internet. Parent-child dyads without an electronic device were mailed an Amazon Fire tablet that they could use to participate in the online sessions. We began enrollment for the face-to-face study in June 2019 and paused data collection in March 2020, due to the COVID-19 pandemic. In October 2020, we began the enrollment for the online study, for which we used Zoom video conferencing. The online data collection is ongoing; for the purposes of the current manuscript, we report on data collected through mid-June 2021.

Table 1 shows demographic characteristics for the faceto-face and online samples. Face-to-face and online parentchild dyads did not significantly differ in child age, gender, ethnicity, race, gestational age, birthweight, parent education, or household income. Children and parents were predominately White and from high-socioeconomic backgrounds, with an average household income of $\$ 115,648.71$ and an average parent education corresponding to a college degree.

\section{Procedure}

For the face-to-face portion of the study, parent-child dyads attended a 3-h laboratory visit. During the laboratory visit, experimenters administered tasks to children, while parents

TABLE 1 | Demographic information for face-to-face $(N=83)$ and online $(N=48)$ samples.

\begin{tabular}{lcc}
\hline & $\begin{array}{c}\text { Face-to-face } \\
\text { M (SD) or } \boldsymbol{n}(\%)\end{array}$ & $\begin{array}{c}\text { Online } \\
\text { M (SD) or } \boldsymbol{n}(\%)\end{array}$ \\
\hline Child age (years) & $4.77(0.48)$ & $5.15(0.48)$ \\
Child gender & & \\
$\quad$ Female & $38(46 \%)$ & $25(53 \%)$ \\
Child hispanic & $7(8 \%)$ & $4(9 \%)$ \\
Child white & $80(96 \%)$ & $45(94 \%)$ \\
Child premature & $29(35 \%)$ & $9(19 \%)$ \\
Birth weight (lbs, oz) & $6.20(2.42)$ & $6.81(1.71)$ \\
Household income (USD) & $113233(67708)$ & $20204(56229)$ \\
Parent education & $3(4 \%)$ & $6(13 \%)$ \\
$\quad$ High school graduate & $5(6 \%)$ & $4(9 \%)$ \\
Some college credit & $10(12 \%)$ & $10(21 \%)$ \\
Associate's degree & $37(45 \%)$ & $25(53 \%)$ \\
$\quad$ Bachelor's degree & $28(34 \%)$ & $38.61(13.04)$ \\
Professional degree & $36.59(4.87)$ & $43(93 \%)$ \\
Parent age (years) & $75(91 \%)$ & $1(2 \%)$ \\
Parent gender & $3(4 \%)$ & $45 \%)$ \\
Female & $77(93 \%)$ &
\end{tabular}

completed questionnaires on a computer in another room. The face-to-face portion of the study included ten standardized neurocognitive assessments and four experimental tasks. The standardized neurocognitive assessments included six subtests from the Wechsler Preschool \& Primary Scale of Intelligence, Fourth Edition (WPPSI-IV; Wechsler, 2012) (block design, bug search, matrix reasoning, information, similarities, and picture memory) and four subtests from the Developmental Neuropsychological Assessment, Second Edition (NEPSY-II; Brooks et al., 2009) (affect recognition, comprehension of instruction, statue, and theory of mind). The three experimental tasks included Give A Number, What's on This Card, and Mental Rotation. Tasks were administered in blocks, and children took breaks in between each block.

For the online portion of the study, parent-child dyads participated in four 45-60-min sessions via Zoom. Using feedback from a focus group with local parents who expressed concern regarding possible screen fatigue, we structured the online portion of the study across four, shorter online sessions rather than one 3 -h session. During the online sessions, children completed most the same standardized neurocognitive assessments, and parents completed the same online questionnaires. In the online portion of the study, parents were asked to complete the same questionnaires on their own time between session 1 and session 4. Four of the neurocognitive assessments (WPPSI-IV block design, give a number, NEPSY-II comprehension of instructions, and NEPSY-II theory of mind) that were administered face-toface could not reliably be administered in an online format for reasons discussed below. The six remaining standardized neurocognitive assessments and two remaining experimental tasks were divided across four online Zoom sessions. Session 1 included WPPSI-IV matrix reasoning, information, and similarities. Session 2 included what's on this card and mental rotation. Session 3 included NEPSY-II affect recognition and statue. Session 4 included WPPSI-IV picture memory. The order of the tasks was the same in both the face-to-face and online sessions, and tasks were administered by the same research assistants.

For the online portion of the study, parents scheduled their four sessions via Calendly (an online scheduling tool), email, or phone. Once parents scheduled their sessions, experimenters provided parent-child dyads with information to prepare them for their first session. This included information on preparing devices and information on dos and don'ts for the four sessions. For example, experimenters stressed the importance of not providing aid or input during the neurocognitive assessments. This also included a link to the informed consent document if the parent did not fill it out prior. The day of session 1, experimenters emailed parents a secured Zoom video conferencing link, for which they were able to attend without needing a Zoom account. Once parent-child dyads logged onto the session, experimenters guided parents on positioning the camera if necessary. With parental consent, all online sessions were recorded through Zoom. The same procedures were followed for session 2 through session 4. Further information on task set up is in Table 2. 
TABLE 2 | Task descriptions for face-to-face and online procedures.

\begin{tabular}{|c|c|c|c|c|}
\hline Task & $\begin{array}{c}\text { Included } \\
\text { face-to-face? }\end{array}$ & $\begin{array}{l}\text { Included } \\
\text { online? }\end{array}$ & $\begin{array}{l}\text { Timing/response } \\
\text { type for online }\end{array}$ & Face-to-face procedure \\
\hline \multicolumn{5}{|l|}{ Verbal comprehension } \\
\hline WPPSI-IV information & $\checkmark$ & $\checkmark$ & $\begin{array}{l}\text { Untimed / verbal } \\
\text { response }\end{array}$ & $\begin{array}{l}\text { For questions involving pictures, } \\
\text { experimenters presented children with } \\
\text { visual stimuli via a testing binder, and } \\
\text { flipped the pages when children were } \\
\text { ready to move items. Children provided } \\
\text { their response by pointing. For questions } \\
\text { involving verbal response, experimenters } \\
\text { read questions aloud, and children } \\
\text { responded verbally. }\end{array}$ \\
\hline
\end{tabular}

Online procedure

\section{Verbal comprehension}

WPPSI-IV information

WPPSI-IV similarities

Language

NEPSY-II comprehension

of instructions

Visuospatial

WPPSI-IV block design

Mental rotation

$\checkmark$

$\checkmark$

Fluid reasoning

WPPSI-IV matrix reasoning

Untimed / verbal response
Untimed / response stamp
Untimed / response stamp
For questions involving pictures, experimenters presented children with visual stimuli via a testing binder, and flipped the pages when children were ready to move items. Children provided their response by pointing. For questions involving verbal response, experimenters read questions aloud, and children responded verbally.
For questions involving pictures, experimenters presented children with visual stimuli via Microsoft PowerPoint; experimenters shared screen and advanced slides when children were ready to move items. Children provided their response by 'stamping' via Zoom's remote-control feature, on the experimenter's screen using a computer mouse or trackpad. Parents were asked to assist, during which they helped their child 'stamp' their response. For questions involving verbal response, participants were administered the Information subtest online using the same method as in-person.

For questions involving pictures, experimenters presented children with visual stimuli via Microsoft PowerPoint; experimenters shared screen and advanced slides when children were ready to move items. Children provided their response by 'stamping' via Zoom's remote-control feature, on the experimenter's screen using a computer mouse or trackpad. Parents were asked to assist, during which they helped their child 'stamp' their response. For questions involving verbal response, participants were administered the Similarities subtest online using the same method as in-person.

Experimenters presented children with visual stimuli via a testing binder, during which experimenters gave verbal instructions that increased in complexity and could not be repeated. Children provided their response by pointing.

Experimenters presented children with blocks of various colors and patterns and asked children to model 3-D block patterns of increased complexity Experimenters presented children with visual stimuli via a testing binder and flipped the pages when children were ready to move items. Children provided their response by pointing.

Untimed / response stamp
Experimenters presented children with visual stimuli via a testing binder and flipped the pages when children were ready to move items. Children provided their response by pointing.
Experimenters presented children with visual stimuli via Microsoft PowerPoint; experimenters shared screen and advanced slides when children were ready to move items. Children provided their response by 'stamping' via Zoom's remote-control feature, on the experimenter's screen using a computer mouse or trackpad. Parents were asked to assist, during which they helped their child 'stamp' their response.

Experimenters presented children with visual stimuli via Microsoft PowerPoint; experimenters shared screen and advanced slides when children were ready to move items. Children provided their response by 'stamping' via Zoom's remote-control feature, on the experimenter's screen using a computer mouse or trackpad. Parents were asked to assist, during which they helped their child 'stamp' their response. 
TABLE 2 | (Continued)

\begin{tabular}{|c|c|c|c|c|c|}
\hline Task & $\begin{array}{c}\text { Included } \\
\text { face-to-face? }\end{array}$ & $\begin{array}{l}\text { Included } \\
\text { online? }\end{array}$ & $\begin{array}{l}\text { Timing/response } \\
\text { type for online }\end{array}$ & Face-to-face procedure & Online procedure \\
\hline \multicolumn{6}{|l|}{ Working memory } \\
\hline WPPSI-IV picture memory & $\checkmark$ & $\checkmark$ & $\begin{array}{c}\text { Timed / response } \\
\text { stamp }\end{array}$ & $\begin{array}{l}\text { Experimenters presented children with } \\
\text { visual stimuli via a testing binder and } \\
\text { flipped the pages when children were ready } \\
\text { to move items. Children provided their } \\
\text { response by pointing. }\end{array}$ & $\begin{array}{l}\text { Experimenters presented children with } \\
\text { visual stimuli via Microsoft PowerPoint; } \\
\text { experimenters shared screen and } \\
\text { advanced slides when children were ready } \\
\text { to move items. Children provided their } \\
\text { response by 'stamping' via Zoom's } \\
\text { remote-control feature, on the } \\
\text { experimenter's screen using a computer } \\
\text { mouse or trackpad. Parents were asked to } \\
\text { assist, during which they helped their child } \\
\text { 'stamp' their response. }\end{array}$ \\
\hline
\end{tabular}

executive functioning

NEPSY-II statue

Social perception

NEPSY-II affect

recognition

NEPSY-II theory of mind

$\checkmark$

Numerical

What's on this card

Untimed / verbal response

Untimed / verbal
response / response

$$
\text { stamp }
$$

ned / response stamp

For the Verbal task and contextual tasks, experimenters presented children with visual stimuli via a testing binder and flipped the pages when children were ready to move items. For the Contextual task, children provided their response by pointing. subtest online using the same method as in-person, with the exception that experimenters often asked parents to help orient their child in the position of the camera. Otherwise, parents were not required to assist on the Statue subtest.

Experimenters presented children with visual stimuli via a testing binder and flipped the pages when children were ready to move items. Children provided their response by pointing.

Experimenters presented children with visual stimuli via a testing binder and flipped the pages when children were ready to move items. Children provided their response by responding verbally.

Experimenters presented children with a pile of fifteen plastic fish, during which experimenters asked children to place a certain number of fish (i.e., 1-9) into a fishbowl.

Processing speed WPPSI-IV bug search

Timed / response stamp
Experimenters presented children with visual stimuli via Microsoft PowerPoint; experimenters shared screen and advanced slides when children were ready to move items. Children provided their response by 'stamping' via Zoom's remote-control feature, on the experimenter's screen using a computer mouse or trackpad. Parents were asked to assist, during which they helped their child 'stamp' their response.

Experimenters presented children with visual stimuli via Microsoft PowerPoint experimenters shared screen and advanced slides when children were ready to move items. Children provided their response by responding verbally. Parents were not required to assist on the WOC task.

\footnotetext{
Experimenters presented children with visual stimuli via a testing packet and flipped the pages when children were ready to move items. Experimenters asked the children to match various kinds of bugs to one another from an assortment of response options using a child-friendly ink dauber, within one minute and 15 seconds.
}

\section{Measures Administered Both Face-to-Face and Online}

Experimenters administered the following measures. It should be noted that publishers of standardized neurocognitive assessments did not provide the online materials; we adapted the materials for online administration for research purposes. This is true for all WPPSI-IV and NEPSY-II materials. The WPPSI-IV and NEPSY-II have been shown to have strong reliability and validity 
when measured in a face-to-face format (Brooks et al., 2009; Wechsler, 2012). We also measured internal consistency using Cronbach's alpha in our two experimental tasks. Reliability across face-to-face and online participants on What's on This Card was good (a > 0.75). Reliability across face-to-face participants on Mental Rotation was acceptable $(a=0.50)$, and across online participants, reliability was good $(a=0.74)$. Please see Table 2 for further details on the tasks referenced below.

\section{Verbal Comprehension WPPSI-IV information}

Experimenters administered the Information subtest, part of the WPPSI-IV Verbal Comprehension Index (VCI). The VCI measures a child's acquired knowledge, verbal reasoning, and comprehension skills. The Information subtest uses both visual and verbal stimuli to assess children's acquired knowledge (e.g., "what do people use to stay dry in the rain?") (Wechsler, 2012).

\section{WPPSI-IV similarities}

Experimenters administered the Similarities subtest, part of the WPPSI-IV VCI. The Similarities subtest asks children, using Picture Tasks and Verbal Tasks, to describe how two words that share a common characteristic are related to one another (e.g., "red and yellow are both...") (Wechsler, 2012).

\section{Fluid Reasoning WPPSI-IV matrix reasoning}

Experimenters administered the Matrix Reasoning (WPPSI-MR) subtest, part of the WPPSI-IV Fluid Reasoning Index (FRI). WPPSI-MR measures visual processing and spatial perception by asking children to select a missing portion from a matrix (Wechsler, 2012).

\section{Visual Spatial \\ Mental rotation}

Experimenters administered a shortened version of the Children's Mental Transformation Task (CMTT, Levine et al., 1999). The Children's Mental Transformation Task is a non-verbal spatial task, during which children are presented with four shapes and two halves of a $2 \mathrm{D}$ shape and asked to select the shape that the two halves would make if they were put together.

\section{Working Memory WPPSI-IV picture memory}

Experimenters administered the Picture Memory (WPPSI-PM) subtest, part of the WPPSI-IV Working Memory Index (WMI). WPPSI-PM measures a child's working memory by asking children to look at pictures of increasingly complex quantities, for three seconds, before asking children to point to those they viewed on a response page (Wechsler, 2012).

\section{Attention and Executive Functioning NEPSY-II statue}

Experimenters administered the Statue subtest, part of the NEPSY-II Attention and Executive Functioning domain, which measures a child's motor persistence and inhibition (Brooks et al., 2009). Experimenters asked children to remain as still as possible, during which experimenters deducted points if children opened their eyes, made drastic body movements, and/or spoke.

\section{Social Perception \\ NEPSY-II affect recognition}

Experimenters administered the affect recognition (AR) subtest, part of the NEPSY-II Social Perception domain. The AR subtest measures a child's ability to recognize affect (Brooks et al., 2009). In four different tasks, experimenters showed children variations of affect from photographs of children's faces, during which experimenters' assessed children' ability to recognize affect between children in each task (Brooks et al., 2009).

\section{Numerical}

\section{What's on this card}

Experimenters administered a What's on This Card task, during which experimenters asked children to vocalize what they saw on twelve different cards. For example, experimenters showed a card with three soccer balls and asked children "what is on this card?" Experimenters scored the total amount of cards the participant responded correctly to, out of a total of seventeen.

\section{Measures Administered Face-To-Face but Not Online}

We were unable to move several face-to-face measures to an online format. Some tasks were excluded because we were not able to provide the necessary materials to each participant. For example, we could not administer the WPPSI-IV block design (BD) subtest, part of the WPPSI-IV visual spatial index (VSI), in an online format because we were unable to supply the standardized materials (i.e., blocks, assessment binder, and stopwatch) to every participant. Likewise, we could not administer WPPSI-IV bug search (BS) subtest, part of the WPPSI-IV processing speed index (PSI), and an experimental numerical task, give a number task, due to the same reason.

Other tasks were excluded because we were concerned about administering these tasks in a standardized fashion across various electronic devices (i.e., laptop vs. tablet) and internet reliabilities. For example, we chose to not administer the NEPSYII comprehension of instructions (CI) subtest, part of the NEPSY-II Language domain, due to being unable to repeat the verbal instructions to the child in case a problem with internet connection occured. We chose to not administer the NEPSYII theory of mind (TM) subtest, part of the NEPSY-II Social Perception domain, due to the same reason.

\section{Parental Involvement Coding}

For online sessions only, we categorized parental involvement into two categories: (1) parent absent or quiet and (2) parent present but with minimal involvement, including directing attention or rewording the instructions. If the parent was present with significant, more than minimal, involvement, the child's score from that task was excluded from the analyses below. Examples of significant involvement included parents providing strategies relevant to the task that would significantly influence child performance or parents simply providing the answer. This 
coding was completed after the sessions were done using the video recordings of the interaction.

\section{Analytic Plan}

We examined whether children's performance varied as a function of format. A sequence of multiple linear regressions were run using the $l m$ function in the stats package (R Core Team, 2013) to determine whether children in the online condition performed differently than children administered the battery face-to-face. Format was dummy coded with face-to-face condition used as the reference group. In predicting performance on WPPSI-IV and NEPSY-II, we controlled for parent education only because we used participant's scaled scores adjusted for age at testing. In predicting performance on What's On This Card and Mental Rotation, we controlled for parent education and child age at testing. For exploratory purposes, we also examined if child performance differed in the face-to-face and online format as a function of prematurity (TB vs. PTB) by later adding the interaction component between prematurity and format to the sequence of multiple linear regressions used to answer research question 1. Finally, again for exploratory analyses, we examined if child performance at a given session varied as a function of parental involvement in the session during which task was administered using $t$-tests with involvement as two categories (no involvement vs. minimal involvement).

\section{RESULTS}

\section{How Do Children's Performance in Verbal Comprehension, Fluid Reasoning, Visual Spatial, Working Memory, Attention and Executive Functioning, Social Perception, and Numerical Skills Differ From Face-to-Face to Online Format?}

Figure 1 and Table 3 represent children's performance on several measures, both in the face-to-face and in the online study. Face-to-face and online samples differed significantly from each other on the following tasks: WPPSI-IV information, WPPSIIV similarities, and WPPSI-IV MR. Scores on all three subtests were reliably lower in the face-to-face sample than in the online sample, when controlling for parent education.

Scores on the following standardized neurocognitive assessments did not differ between children who participated face-to-face and children who participated online, when controlling for parent education: WPPSI-IV PM, NEPSY-II AR, and NEPSY-II statue. Scores on the following experimental tasks did not differ between children who participated face-toface and children who participated online, when controlling for parent education and age at testing: mental rotation and what's on this card.

It could be the case that the lowest performing children were unable to perform the tasks online, resulting in experimenters dropping their scores. To test this possibility, we conducted a follow-up analysis, in which we compared whether the number of children whose data were dropped in the face-to-face vs. online study differed from each other. Data were excluded for the following reasons: significant parent involvement, child fatigue, and experimenter error. The number of children whose data were included vs. excluded from the analysis are reported in Table 3 and were compared across the two formats using ChiSquare analyses. None of the comparisons reached statistical significance (all $p$ 's $>0.05$ ), suggesting that number of children whose data were excluded due to reasons stated above did not vary across formats.

\section{What Is the Role of Parental Involvement in Children's Performance in the Online Format?}

Finally, for the children who participated in the online format only, we examined if parent involvement played a role. To reduce the number of analyses we ran, we only conducted these exploratory analyses on the three WPPSI-IV subtests on which children performed better in the online format as compared to face-to-face format. For children whose data were included in the first session, twenty-five parents were not involved and 22 were minimally involved. $t$-test analyses comparing children of parents who were not involved vs. those who were minimally involved did not reveal any significant differences on WPPSIIV similarities, $t(42)=0.08, p=0.94$, WPPSI-IV information, $t(43)=2.02, p=0.36$, or on WPPSI-IV MR, $t(39)=1.23, p=0.23$.

\section{How Do Children's Performance in Verbal Comprehension, Fluid Reasoning, Visual Spatial, Working Memory, Attention and Executive Functioning, Social Perception, and Numerical Skills Differ From Face-to-Face to Online Format as a Function of Prematurity?}

Scores on all standardized neurocognitive assessments did not differ between children who participated face-to-face and children who participated online as a function of prematurity, except on WPPSI-IV MR. A reliable bivariate interaction emerged between format and prematurity on WPPSI-IV MR, $t(114)=-2.22, p<0.05$. PTB children performed lower than TB children in the online format, as compared to children in the face-to-face format.

\section{DISCUSSION}

The COVID-19 pandemic has catalyzed an increasing interest in the best practices for online data collection methods in developmental science. As COVID-19 restrictions persist, remote methods will be paramount to developmental science research. Here, we aimed to contribute to the discussions on online data collection methods. Specifically, we asked whether children who participated in study visits face-to-face and children who participated in study visits online performed differently on both standardized and experimental measures. We examined this question in both typically developing, term-born (TB) children, 


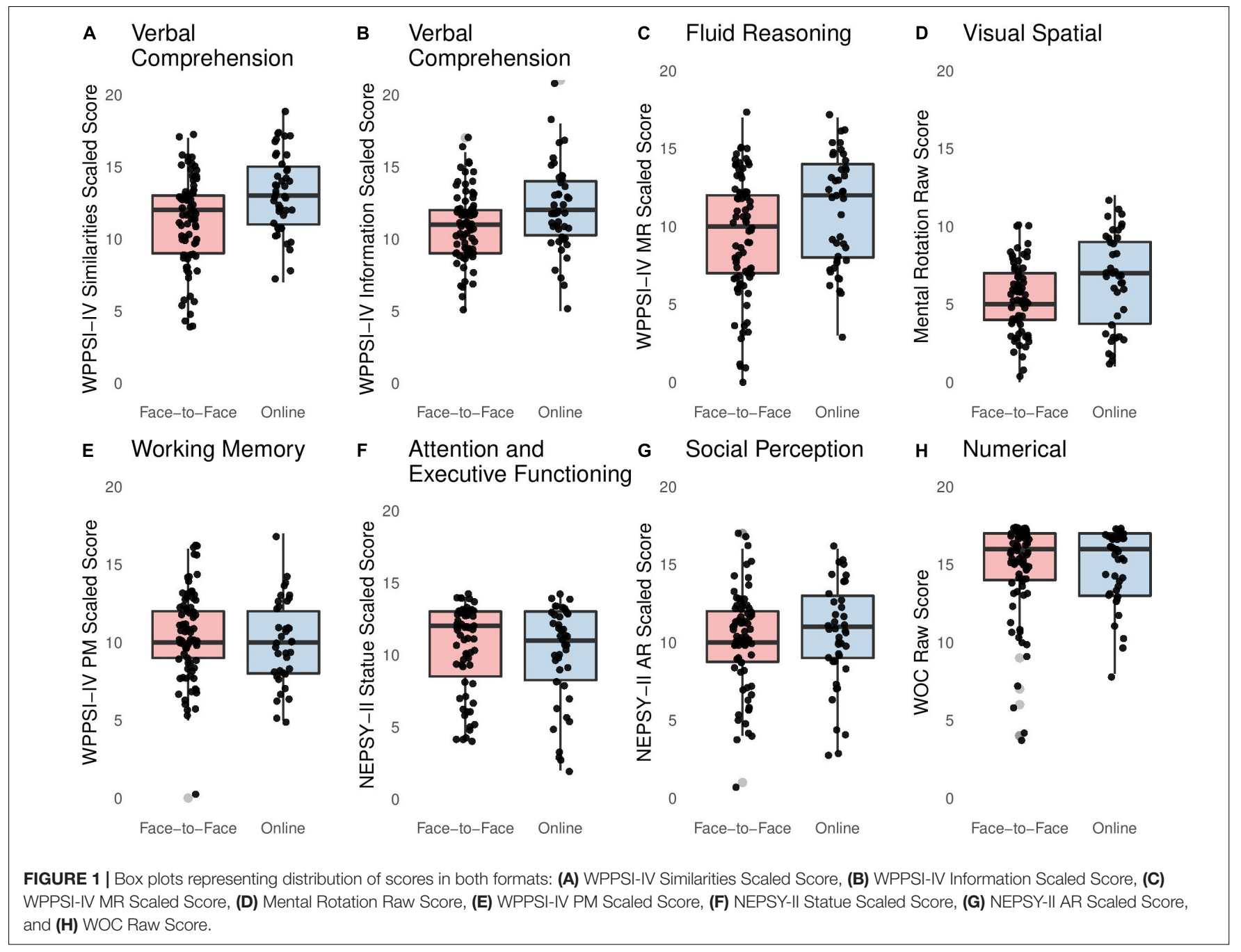

TABLE 3 | Results of mean scores captured in both formats, participants included and excluded, and regression analyses comparing format controlling for parent education and child age at testing.

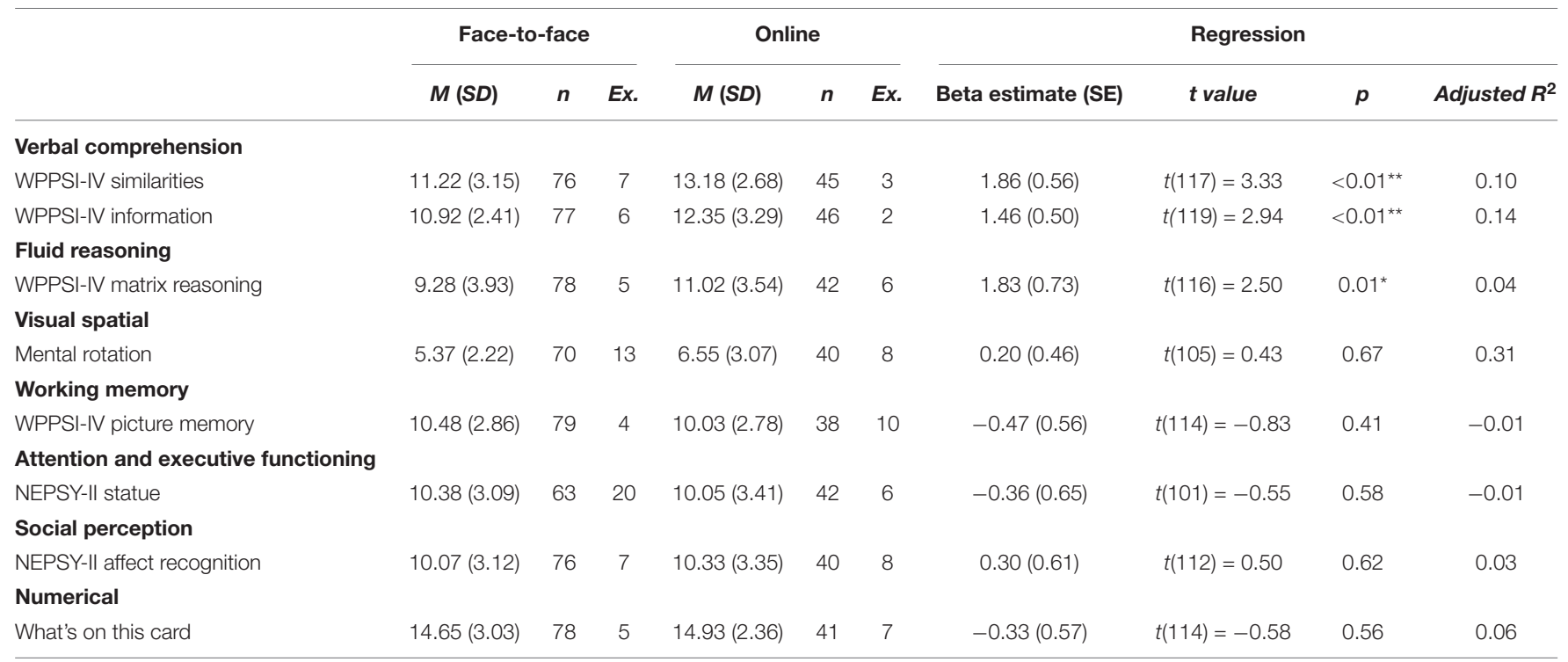

Statistics represent beta estimate, standard error, $T$ Value, and $P$ value for the Effect of Format. Ex., excluded. ${ }^{*} p<0.05 ;{ }^{* *} p<0.01$. 
and in at-risk, preterm-born (PTB) children. We also explored whether parental involvement in the online format related to children's performance.

The finding that children's performance did not vary across the two formats for most of the measures that we administered provides support for the utilization of online data collection methods in developmental science. Here, we provide empirical evidence suggesting that children's performance was not significantly influenced by format on a wide range of cognitive assessments, both standardized and experimental. Our results may help alleviate some of the concerns that researchers have raised about online data collection methods. First, researchers have expressed concern about having less control over the testing environment and having a higher number of distractions, which might lead to a greater portion of data being excluded from online formats. However, we examined this hypothesis, and this was not the case in our sample. It is important to highlight that, in both formats, trained clinical science graduate students audited study visits and excluded data that was thought to be an inaccurate representation of the child's performance. For example, if it was clear that the child was not answering questions due to shyness, that child's data was excluded. Second, there has been concern about differences in sample characteristics between samples that participate face-to-face and samples that participate online, including concerns about differences in demographic characteristics (e.g., parental education) and in health characteristics (e.g., children with special health needs). In our sample, group comparisons did not reveal any significant sociodemographic differences between the two groups. It should be noted that the average income and average education of our sample were generally high, so differences might emerge in a more socioeconomically diverse sample.

Moreover, we were able to recruit PTB children, who are atrisk for academic challenges. Although we were not sufficiently powered to include analyses on interactions between prematurity and format, we conducted exploratory analyses examining such interactions. Reliable bivariate interactions between format and prematurity did not emerge on any of the tests, except for WPPSIIV MR; PTB children performed lower than TB children in the online format, but not face-to-face format. Thus, overall, format did not appear to greatly undermine preterm children's performance. Future studies that are sufficiently powered should examine interactions between format and risk factors.

While children's performance did not vary by format on most of the assessments, their scores on WPPSI-IV information, similarities, and matrix reasoning did. Average scores on these three subtests were lower for those who participated in the faceto-face format. This finding is inconsistent with findings from a previous study, suggesting equivalence in online and face-toface scores on information, similarities, and matrix reasoning (Wright, 2020). However, this study included older children and leveraged proctors instead of parents. Thus, it is possible that parents played a role in our findings. However, our analyses showed that children who had more parental involvement and children who had no parental involvement did not differ on information, similarities or matrix reasoning. Another possibility is that the online format lent itself to performing better, due to children participating in the study in the comfort their own homes. During face-to-face study visits, children completed testing in an unfamiliar lab while their parents were in another room; during online study visits, children completed testing in their homes while their parents were sitting next to them. While we might expect to see this increased comfort reflected in other tests as well, comfort at the beginning of the study might have differed to a greater extent across the two formats. Information, similarities, and matrix reasoning were administered during the first part of the study visit in both formats. However, in the online format, parents and children played together for 5-10 $\mathrm{min}$ prior to the tasks. Although the children were familiarized with the research assistant via play in the lab as well, playing with specifically the parent in the online format could have made children feel more comfortable prior to testing. Finally, it is possible that those who participated online were exposed to factors, whether related to the pandemic or not, that those who participated face-to-face were not. Maybe those who participated online had experiences that benefited their performance on information, similarities, and matrix reasoning. For example, it is possible that those who participated online spent more time interacting with their parents than those who participated faceto-face, due to parents being at home more during the pandemic. Indeed, the verbal skills and acquired knowledge involved in information and similarities, for example, may be sensitive to parental input (Kan et al., 2011; Pace et al., 2016). However, the other tasks included in this study may also benefit from parental input (Clingan-Siverly et al., 2021). Thus, future research should explore whether different types of parental input influenced some cognitive abilities but not others.

Our study has limitations that should be discussed for future studies. First, children may have performed better in the online format due to ordering effects. We did not counterbalance the standardized neurocognitive assessments and experimental tasks for the face-to-face portion of the study. Therefore, we stayed consistent when structing the online portion of the study and continued with the same order of administration. Future research would benefit from counterbalancing the battery of neurocognitive assessments. These neurocognitive assessments demand a lot of attention and children can easily become fatigued throughout the span of assessments. Second, our combined sample was predominately $\mathrm{TB}$ children, compared to PTB children. Future research would benefit from having balanced numbers of TB and PTB children. Third, our combined sample was also predominately of a high socioeconomic status. Future research would benefit from having a more diverse sample, so we could better generalize our findings. Last, due to the small sample size, especially in the online group, we may be underpowered to detect main, and more likely, interaction effects. Thus, we argue that our findings should be replicated in future studies with larger samples.

Taken together, our results suggest that online data collection might be a feasible option for several cognitive measures, for both PTB and TB children. Our results also suggest that, however, online data collection for certain measures, including WPPSI-IV information, similarities, and matrix reasoning should be interpreted with caution. Future research should examine 
the mechanisms through which data collection format might influence children's performance. Relevant factors to consider include parental involvement and familiarity with the setting. In addition to our empirical findings, we demonstrated success in recruiting and including several families from lower-resourced rural communities and several families with preterm children to participate online, highlighting the feasibility of including samples with different demographic and health characteristics when using online methods. This not only has implications for research methods but also for providing prevention and intervention services. Our results contribute to the growing body of literature examining differences and similarities across various data collection methods. Online data collection may be a good option for continuing developmental psychology research, for diversifying research samples, and for providing services (e.g., educational services and clinical services) when traditional methods are not available. Our findings can also inform future studies hoping to explore the use of online test administration for educational and clinical purposes, as online methods may be more convenient and accessible for both providers and families.

\section{DATA AVAILABILITY STATEMENT}

The raw data supporting the conclusions of this article will be made available by the authors, without undue reservation.

\section{ETHICS STATEMENT}

The studies involving human participants were reviewed and approved by University of Iowa Institutional Review

\section{REFERENCES}

Allotey, J., Zamora, J., Cheong-See, F., Kalidindi, M., Arroyo-Manzano, D., Asztalos, E., et al. (2018). Cognitive, motor, behavioural and academic performances of children born preterm: a meta analysis and systematic review involving 64061 children. BJOG 125, 16-25. doi: 10.1111/1471-0528. 14832

Bhutta, A. T., Cleves, M. A., Casey, P. H., Cradock, M. M., and Anand, K. J. (2002). Cognitive and behavioral outcomes of school-aged children who were born preterm: a meta-analysis. JAMA 288, 728-737. doi: 10.1001/jama.288.6.728

Brooks, B. L., Sherman, E. M., and Strauss, E. (2009). NEPSY-II: a developmental neuropsychological assessment. Child Neuropsychol. 16, 80-101. doi: 10.1080/ 09297040903146966

Brydges, C. R., Landes, J. K., Reid, C. L., Campbell, C., French, N., and Anderson, M. (2018). Cognitive outcomes in children and adolescents born very preterm: a meta analysis. Dev. Med. Child Neurol. 60, 452-468. doi: 10.1111/dmcn.13685

Bureau, U. S. C. (2021). Nearly 93\% of Households With School-Age Children Report Some Form of Distance Learning During COVID-19. Census.gov. Availble online at: https://www.census.gov/library/stories/2020/08/schoolingduring-the-covid-19-pandemic.html (accessed October 15, 2021).

Centers for Disease Control and Prevention [CDC] (2020). Preterm Birth. Available online at: https://www.cdc.gov/reproductivehealth/maternalinfanthealth/ pretermbirth.htm (accessed April 15, 2021).

Clingan-Siverly, S., Nelson, P. M., Göksun, T., and Demir-Lira, ÖE. (2021). Spatial thinking in term and preterm-born preschoolers: relations to parent-child speech and gesture. Front. Psychol. 12:651678. doi: 10.3389/fpsyg.2021.651678
Board. Written informed consent to participate in this study was provided by the participants' legal guardian/next of kin.

\section{AUTHOR CONTRIBUTIONS}

ÖD-L, PN, and FS conceptualized the study and wrote the manuscript. PN, FS, and HL collected the data. ÖD-L and PN analyzed the data. All authors prepared the data for analysis and contributed to the article and approved the submitted version.

\section{FUNDING}

This research was supported by NICHD R03HD102449 and the Centers for Disease Control and Prevention of the U.S. Department of Health and Human Services (HHS) as part of a Cooperative Agreement Number (U48 DP006389). FS was supported by the NIHGMS grant T32GM108540. The contents are those of the author(s) and do not necessarily represent the official views of, nor an endorsement, by CDC/HHS, or the U.S. Government.

\section{ACKNOWLEDGMENTS}

The authors would like to thank the families who participated in our study and research assistants who helped with data collection.

EducationData (2021). Research and Resources to Tackle Critical Issues in Education. Available online at: https://educationdata.org/online-educationstatistics (accessed April 15, 2021).

Frank, M. C., Sugarman, E., Horowitz, A. C., Lewis, M. L., and Yurovsky, D. (2016). Using tablets to collect data from young children. J. Cogn. Dev. 17, 1-17. doi: 10.1080/15248372.2015.1061528

Garrisi, K., King, C. J., Hillyer, L., and Gaab, N. (2020). General Recommendations and Guidelines for Remote Assessment of Toddlers and Children. doi: 10.31219/ osf.io/wg4ef

Kan, K. J., Kievit, R. A., Dolan, C., and van der Maas, H. (2011). On the interpretation of the CHC factor Gc. Intelligence 39, 292-302. doi: 10.1016/j. intell.2011.05.003

Levine, S. C., Huttenlocher, J., Taylor, A., and Langrock, A. (1999). Early sex differences in spatial skill. Dev. Psychol. 35, 940-949. doi: 10.1037/0012-1649. 35.4.940

Lourenco, S. F., and Tasimi, A. (2020). No participant left behind: conducting science during COVID-19. Trends Cogn. Sci. 24, 583-584. doi: 10.1016/j.tics. 2020.05.003

Manning, B. L., Harpole, A., Harriott, E. M., Postolowicz, K., and Norton, E. S. (2020). Taking language samples home: feasibility, reliability, and validity of child language samples conducted remotely with video chat versus in-person. J. Speech Lang. Hear. Res. 63, 3982-3990. doi: 10.1044/2020_JSLHR-20-00202

Morini, G., and Blair, M. (2021). Webcams, songs, and vocabulary learning: a comparison of in-person and remote data collection as a way of moving forward with child-language research. Front. Psychol. 12:702819. doi: 10.3389/fpsyg. 2021.702819 
Nussenbaum, K., Scheuplein, M., Phaneuf, C. V., Evans, M. D., and Hartley, C. A. (2020). Moving developmental research online: comparing in-lab and webbased studies of model-based reinforcement learning. Collabra Psychol. 6:17213. doi: 10.1525/collabra.17213

Pace, A., Levine, D., Morini, G., Hirsh-Pasek, K., and Golinkoff, R. M. (2016). “The story of language acquisition: from words to world and back again," in Child Psychology: A Handbook of Contemporary Issues, 3rd Edn, eds. L. Balter and C. Tamis-LeMonda 43-79.

R Core Team (2013). R: A Language and Environment for Statistical Computing. Vienna: R Foundation for Statistical Computing.

Rhodes, M., Rizzo, M. T., Foster-Hanson, E., Moty, K., Leshin, R. A., Wang, M., et al. (2020). Advancing developmental science via unmoderated remote research with children. J. Cogn. Dev. 21, 477-493. doi: 10.1080/15248372.2020. 1797751

Sheskin, M., Scott, K., Mills, C. M., Bergelson, E., Bonawitz, E., Spelke, E. S., et al. (2020). Online developmental science to foster innovation, access, and impact. Trends Cogn. Sci. 24, 675-678. doi: 10.1016/j.tics.2020. 06.004

Snijders, V. E., Bogicevic, L., Verhoeven, M., and van Baar, A. L. (2020). Toddlers' language development: the gradual effect of gestational age, attention capacities, and maternal sensitivity. Int. J. Environ. Res. Public Health 17:7926. doi: 10. 3390/ijerph17217926

Su, I. A., and Ceci, S. (2021). "Zoom Developmentalists": home-based videoconferencing developmental research during COVID-19. PsyArXiv [Preprint]. doi: 10.31234/osf.io/nvdy6

Tsega, M., Giantris, K., and Shah, T. (2020). Essential Social Services are Struggling to Survive the COVID-19 Crisis. New York, NY: The Commonwealth Fund.
United Nations Children's Fund (2021). COVID-19: Schools for more than 168 Million Children Globally have been Completely Closed for Almost a Full Year, says UNICEF. New York, NY: UNICEF.

Wechsler, D. (2012). Wechsler Preschool and Primary Scale of Intelligence, 4th Edn. San Antonio, TX: The Psychological Corporation.

Wolke, D., Johnson, S., and Mendonça, M. (2019). The life course consequences of very preterm birth. Annu. Rev. Dev. Psychol. 1, 69-92. doi: 10.1146/annurevdevpsych-121318-084804

Wright, A. J. (2020). Equivalence of remote, digital administration and traditional, in-person administration of the Wechsler Intelligence Scale for Children, (WISC-V). Psychol. Assess. 32, 809-817. doi: 10.1037/pas0000939

Conflict of Interest: The authors declare that the research was conducted in the absence of any commercial or financial relationships that could be construed as a potential conflict of interest.

Publisher's Note: All claims expressed in this article are solely those of the authors and do not necessarily represent those of their affiliated organizations, or those of the publisher, the editors and the reviewers. Any product that may be evaluated in this article, or claim that may be made by its manufacturer, is not guaranteed or endorsed by the publisher.

Copyright (c) 2021 Nelson, Scheiber, Laughlin and Demir-Lira. This is an open-access article distributed under the terms of the Creative Commons Attribution License (CC BY). The use, distribution or reproduction in other forums is permitted, provided the original author(s) and the copyright owner(s) are credited and that the original publication in this journal is cited, in accordance with accepted academic practice. No use, distribution or reproduction is permitted which does not comply with these terms. 